\title{
Topographic voltage and coherence mapping of brain potentials by means of the symbolic resonance analysis
}

\author{
Peter beim Graben, ${ }^{1,2, *}$ Stefan Frisch, ${ }^{1,3,4}$ Andrew Fink, ${ }^{1,2}$ Douglas Saddy, ${ }^{1}$ and Jürgen Kurths ${ }^{2}$ \\ ${ }^{1}$ Institute of Linguistics, Universität Potsdam, P.O. Box 601553, 14415 Potsdam, Germany \\ ${ }^{2}$ Institute of Physics, Nonlinear Dynamics Group, Universität Potsdam, P.O. Box 601553, 14415 Potsdam, Germany \\ ${ }^{3}$ Day-Care Clinic of Cognitive Neurology, Universität Leipzig, Liebigstraße 22a, 04103 Leipzig, Germany \\ ${ }^{4}$ Max-Planck Institute for Human Cognitive and Brain Sciences, P.O. Box 500355, 04303 Leipzig, Germany
}

(Received 15 June 2005; published 11 November 2005)

\begin{abstract}
We apply the recently developed symbolic resonance analysis to electroencephalographic measurements of event-related brain potentials (ERPs) in a language processing experiment by using a three-symbol static encoding with varying thresholds for analyzing the ERP epochs, followed by a spin-flip transformation as a nonlinear filter. We compute an estimator of the signal-to-noise ratio (SNR) for the symbolic dynamics measuring the coherence of threshold-crossing events. Hence, we utilize the inherent noise of the EEG for sweeping the underlying ERP components beyond the encoding thresholds. Plotting the SNR computed within the time window of a particular ERP component (the N400) against the encoding thresholds, we find different resonance curves for the experimental conditions. The maximal differences of the SNR lead to the estimation of optimal encoding thresholds. We show that topographic brain maps of the optimal threshold voltages and of their associated coherence differences are able to dissociate the underlying physiological processes, while corresponding maps gained from the customary voltage averaging technique are unable to do so.
\end{abstract}

DOI: 10.1103/PhysRevE.72.051916

PACS number(s): 87.80.Tq, 05.50. $+\mathrm{q}, 05.45 .-\mathrm{a}$

\section{INTRODUCTION}

Noise is commonly regarded as being detrimental in analyzing natural or experimental data. Therefore, a bulk of filters and denoising algorithms have been discussed in the literature for the analysis of time series [1-5]. On the other hand, noise may play a constructive role in nonlinear dynamical systems, leading, e.g., to the phenomena of stochastic resonance [6-8] or coherence resonance [9]. Recently, we have suggested a nonlinear data analysis technique basing on the symbolic dynamics of a symmetric threshold crossing detector (cf. [10]), where additive noise of a critical amplitude entails such a resonance effect [11].

Coping with noise is particularly a problem with physiological time series, such as the electroencephalogram (EEG) or event-related brain potentials (ERPs) [12], since these are typically noisy, nonstationary, and exhibit extensive variability $[4,5,13]$. In order to reduce the portion of noise that contaminates ERP phenomena, researchers usually collect an ensemble of EEG trials (the so-called epochs) that are timelocked to the perception or processing of some critical stimuli and compute the average of the ensemble across all measured trials, assuming that the background EEG, considered to be unwanted noise, will thus be canceled out. The resulting averaged potentials exhibit certain peaks, the socalled ERP components, which are labeled by their polarity ("P" for a positive peak; "N" for a negative one) and by their latency time (for a critical review of this method, see [14]). Like other linear methods of data analysis such as power spectra or correlation functions, averaging fails to discriminate between amplitude and phase information [15]. An ERP

*Electronic address: peter@ling.uni-potsdam.de component may therefore reflect either a highly coherent signal of comparatively low amplitude, or, by contrast, a less coherent signal with high-amplitude fluctuations. Thus, due to the blending of coherence and amplitude information that occurs through averaging, signals generated by different physiological processes may be rendered indistinguishable with the averaging technique.

In the neuroscience of language, ERP components are paradigmatically interpreted as being correlated with processing problems based on different types of linguistic information. The N400 component, a negative deflection in the ERP around $400 \mathrm{~ms}$ after the onset of a critical stimulus, has been shown to reflect semantic integration problems. For example, Kutas and van Petten [16] reported such an effect on the word rocks in a sentence such as Mary drinks a glass of rocks. In this example, rocks renders the sentence semantically anomalous (that is, nonsensical), since rocks cannot be drunk.

In this paper, we present a typical language processing experiment in which two linguistically different types of stimuli invoke N400 ERP components that cannot be distinguished by means of the traditional voltage averaging analysis. We demonstrate that the symbolic resonance analysis (SRA, $[11,17]$ ) is able to distinguish between the two conditions by separating voltage and coherence information of the ERP into two distinct topographic representations. Using this technique, the background EEG superimposed with the ERP is regarded not simply as detrimental noise which has to be eliminated, but, by contrast, as a helpful mean to drive a subthreshold ERP signal across the encoding thresholds of a symbolic dynamics with three symbols [11]. The phase transitions of this symbolic dynamics considered as a $(1+1)$-dimensional Potts spin lattice [18] correspond to the instances of stochastic resonance in the threshold system. We 
show that a mean-field spin-flip filter of the Potts model transforms the three-symbol statistics into a distribution of two symbols, from which running cylinder entropies [14] and an estimator of the signal-to-noise ratio (SNR) [19] can be computed. Plotting the SNR against the varying encoding thresholds yields characteristic resonance curves for the data of the two experimental conditions. Those thresholds that lead to the largest differences between the SNR of the experimental conditions, the optimal thresholds, are then presented as topographic voltage brain maps. Additionally, the differences of time-averaged cylinder entropies between conditions for these optimal thresholds give rise to a topographic representation of the coherence differences. We will so demonstrate that the SRA is able to separate voltage and coherence information into two distinct dimensions, thereby opening the possibility to dissociate physiologically different processes by means of these topographic brain mappings.

\section{THE ERP EXPERIMENT}

In a German language processing experiment, Friederici and Frisch [20] found an N400 component on the critical word, the verb (underlined below), in sentences such as (ii) and (iii), compared to correct sentences such as (i). Literal English paraphrases are given below each sentence example.

(i) Correct sentence:

Anna weiß, dass der Kommissar den Banker abhörte ... Anna knows that the inspector the banker monitored ... "Anna knows that the inspector monitored the banker"

(ii) Semantic violation

Anna weiß, dass der Kommissar den Banker abbeizte ... Anna knows that the inspector the banker stained ...

"Anna knows that the inspector stained the banker"

(iii) Argument structure violation

Anna weiß, dass der Kommissar den Banker abreiste ... Anna knows that the inspector the banker departed ...

"Anna knows that the inspector departed the banker"

Both sentences (ii) and (iii) are semantically anomalous. However, in (ii) this is due to the fact that people (such as bankers) cannot be stained, whereas other objects can. In (iii), by contrast, the problem is that no object (whatever its properties are) can be departed, so that the semantic anomaly is in some sense more basic.

Each sentence was presented visually and phrase-byphrase on a computer monitor in a randomized order to each subject while the subject's electrophysiological activity was recorded. After each sentence, a judgment had to be given whether the sentence was acceptable or not. Additional manipulations were included in the experimental material in order to control for possible confounds. The raw EEG data were acquired by means of $64 \mathrm{Ag} / \mathrm{AgCl}$ electrodes with 250 $\mathrm{Hz}$ sampling frequency. They were re-referenced with linked mastoids offline. Only correctly performed and artifact-free trials were included in the analysis; for further technical details see [20]. Customary ERPs were then averaged on the critical word (verb, underlined in the above examples) over 40 trials in each of the three conditions [(i), (ii), and (iii)] over a sample of 16 subjects.

Approximately between 300 and $600 \mathrm{~ms}$ after the occurrence of the critical word on the screen, the voltage average

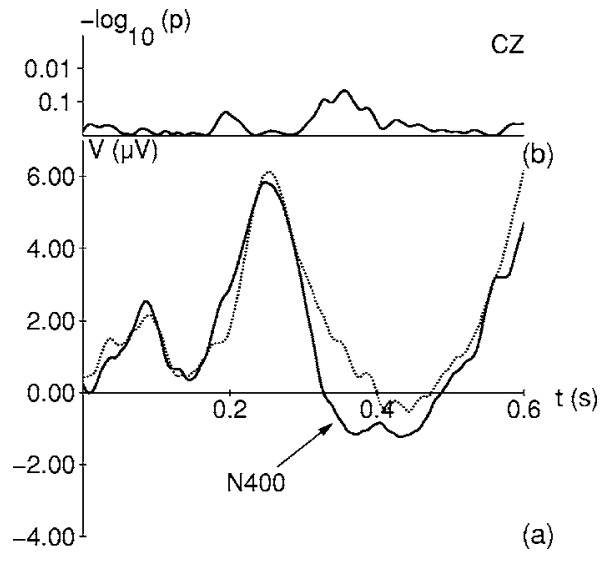

FIG. 1. (a) ERP voltage averages for the conditions (ii) (solid) and (iii) (dotted) at channel CZ (centro-medial). Time onset of the critical stimulus (the verb) at $0 \mathrm{~s}$. The N400 ERP component for both conditions is indicated by the arrow. (b) The error probability of the first kind (in logarithmic scale) of a running $t$-test between conditions (ii) and (iii).

ERPs revealed a negative going wave form, an N400, in both mismatching conditions (ii) and (iii) compared to the correct condition (i). The two violation conditions, (ii) and (iii) differed in that the latter produced a subsequent positive deflection (P600) in addition. However, the N400 negativities in both these conditions were indistinguishable by means of the traditional voltage averaging analysis. The authors were therefore unable to confirm empirically interesting distinctions that might be drawn from linguistic theory.

For the present purpose, we confine ourselves to the N400 component and to the two violation conditions, thereby neglecting the comparison to the correct condition (i). As the N400 is mostly pronounced at centro-parietal electrode sites at the scalp, Fig. 1(a) shows the ERP waves for both conditions (ii) and (iii) at channel CZ. The time onset of the critical stimulus, i.e., the verb, is at $0 \mathrm{~ms}$. The arrow indicates the $\mathrm{N} 400$ as a maximal negative voltage deflection around 400 ms. In order to assess the statistical significance of the ERP difference between both conditions, a running betweensubject $t$-test of the voltage averages has been performed. Figure 1(b) displays its error probability in a logarithmic scale. We see that the difference in the ERPs is obviously not significant at the $5 \%$ error level $(p \approx 0.1)$.

A similar finding is provided by a topographic brain map of the differences of the voltage averaged ERPs from conditions (ii) and (iii) which have been additionally averaged within a time window from $t_{\mathrm{on}}=300 \mathrm{~ms}$ to $t_{\mathrm{off}}=600 \mathrm{~ms}$. This is presented in Fig. 2. We find no significant differences between both conditions by means of a between-subject $t$-test of the voltage averages at the 5\% level.

\section{THE SYMBOLIC RESONANCE ANALYSIS}

In order to dissociate the two N400 components, we analyze the time series from the EEG experiment [20] using our symbolic resonance analysis, which relies upon the cooperative interplay between noise and the nonlinear threshold 


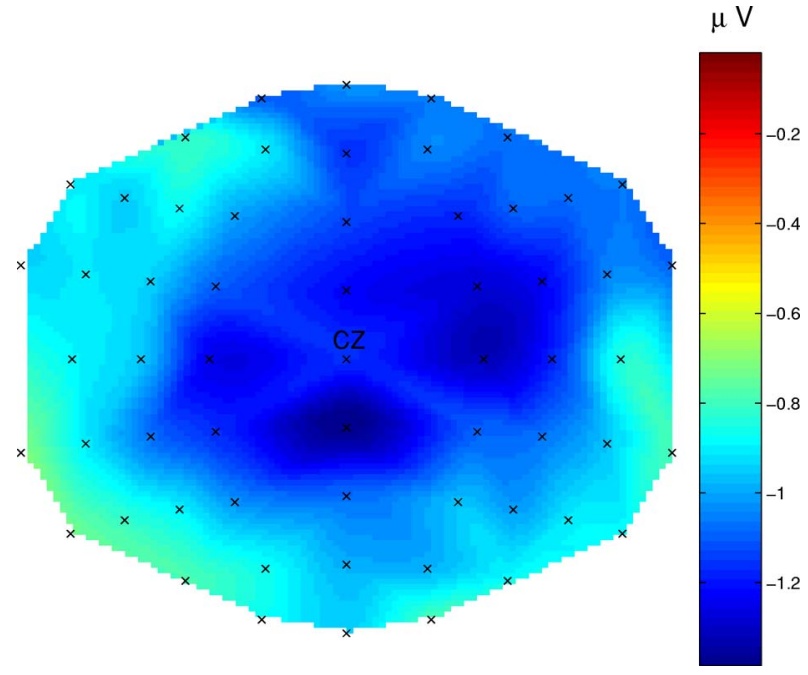

FIG. 2. (Color online) Topographic brain map of the ERP voltage averages further averaged over a time window from 300-600 ms. No significant differences based on a between-subject $t$-test were found. The nose is at the top. Additionally, the channel $\mathrm{CZ}$ is marked.

crossing detector of a symbolic dynamics with three symbols [11]. To do this we use the same raw EEG time series as for the voltage averaging [20]. Each EEG epoch $x(t)$ is first encoded in sequences of the three symbols- $a_{0}=$ " 0 " $[x(t)<-\theta], a_{1}=" 1 "[|x(t)| \leqslant \theta]$, and $a_{2}=$ " 2 " $[x(t)>\theta]$-after aligning their baselines to the time average of a $200 \mathrm{~ms}$ prestimulus interval. The encoding thresholds $\theta$ are tuned from 0.5 to $12.9 \mu \mathrm{V}$ in steps of $0.1 \mu \mathrm{V}$. Afterwards, the symbolic sequences $s=\left(a_{i_{k}}\right)_{1 \leqslant k \leqslant L}$ of all subjects per threshold and per condition are swept up to the grand epoch ensembles

$$
E^{\left(c_{j}\right)}=\left\{s_{i}^{\left(c_{j}\right)} \mid s_{i}^{\left(c_{j}\right)} \in A^{L}, 1 \leqslant i \leqslant N^{\left(c_{j}\right)}\right\}
$$

where the superscripts $\left(c_{j}\right)(j=1,2)$ refer to the experimental conditions. $L$ is the number of sampling points in time, $A=\{$ " $0, "$ " 1, , " 2, , $\}$ the "alphabet" and $N^{\left(c_{j}\right)}$ the number of trials per condition $c_{j}$ (cf. [14], Eqs. (19) and (20)). From these ensembles, the relative frequencies of the symbols in each time slice

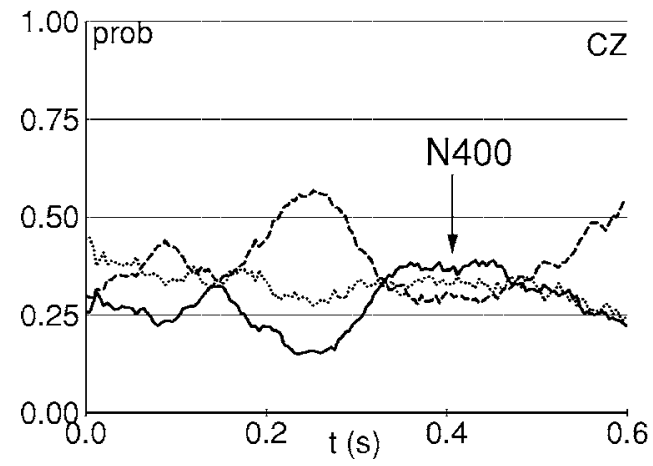

$$
p_{i}^{\left(c_{j}\right)}(t)=\frac{\#\left(a_{i}^{\left(c_{j}\right)}\right)}{N^{\left(c_{j}\right)}}
$$

are determined (see [14], Eq. (21)). These three-symbol distributions are subjected to a nonlinear filter where the meanfields of two competing Potts spins

$$
\begin{aligned}
& M_{0}^{\left(c_{j}\right)}(t)=p_{0}^{\left(c_{j}\right)}(t)-p_{1}^{\left(c_{j}\right)}(t), \\
& M_{2}^{\left(c_{j}\right)}(t)=p_{2}^{\left(c_{j}\right)}(t)-p_{1}^{\left(c_{j}\right)}(t),
\end{aligned}
$$

try to flip the "undecided" between-threshold symbol " 1 " either into " 0 " or into " 2 " leading to a distribution of only two symbols:

$$
\begin{gathered}
p_{0}^{\prime\left(c_{j}\right)}(t)= \begin{cases}p_{0}^{\left(c_{j}\right)}(t), & M_{2}^{\left(c_{j}\right)}(t)>0 \geqslant M_{0}^{\left(c_{j}\right)}(t) \\
p_{0}^{\left(c_{j}\right)}(t)+p_{1}^{\left(c_{j}\right)}(t), & M_{2}^{\left(c_{j}\right)}(t) \leqslant 0<M_{0}^{\left(c_{j}\right)}(t) \\
p_{0}^{\left(c_{j}\right)}(t)+p_{1}^{\left(c_{j}\right)}(t) / 2, & \text { otherwise }\end{cases} \\
\qquad p_{1}^{\prime\left(c_{j}\right)}(t)=1-p_{0}^{\prime\left(c_{j}\right)}(t) .
\end{gathered}
$$

Note that we use a slightly modified definition of the mean-fields compared to that given in [11] [Eq. (5)] in order to emphasize the competition between the " 0 "s and the " 2 "s for the " 1 "s.

Figure 3(a) displays the three-word statistics of condition (ii) at channel $\mathrm{CZ}$ for the encoding threshold $\theta^{\#}=4.2 \mu \mathrm{V}$ (which we will show to be the optimal threshold). The N400 is reflected by the largest frequency of " 0 "s around $400 \mathrm{~ms}$ after stimulus onset, representing negative below threshold voltages. By contrast, Fig. 3(b) illustrates the effect of the filter by showing the two-word statistics resulting from the spin-flip transform. In the $400 \mathrm{~ms}$ window the betweenthreshold symbols " 1 " are completely reverted into " 0 "s due to the impact of the mean-fields. Hence, the N400 corresponds to a highly degenerated word statistics of almost constant amplitude over the characteristic time window.

In order to perform the SRA, we need to compute the estimator of the SNR ([11], Eq. (4)) depending on the noise strength of the EEG. Since the noise itself is not accessible in single EEG epochs, we must systematically vary the encoding thresholds. The first step towards determining the SNR is computing the running cylinder entropies

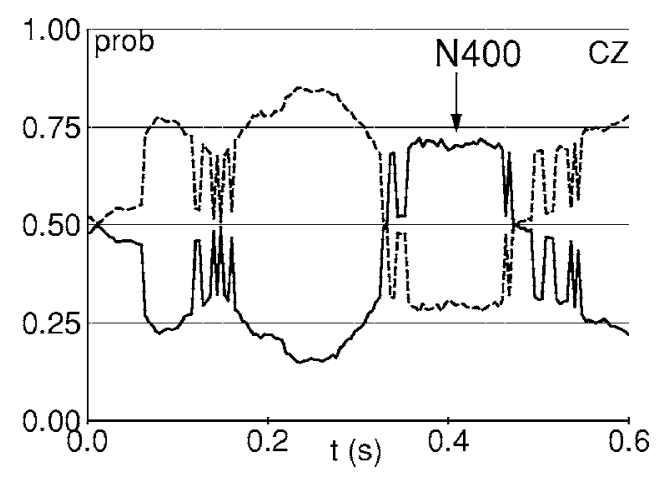

FIG. 3. Word statistics [Eq. (2)] for condition (ii) computed for the optimal encoding threshold $\theta^{\#}=4.2 \mu \mathrm{V}$ [Eq. (10)] at channel CZ. (a) Relative frequencies of the three symbols " 0 " (solid), "1" (dotted), and "2" (dashed). (b) Relative frequencies of the two symbols "0" (solid) and "1" (dashed) after mean-field transform [Eq. (5)]. 


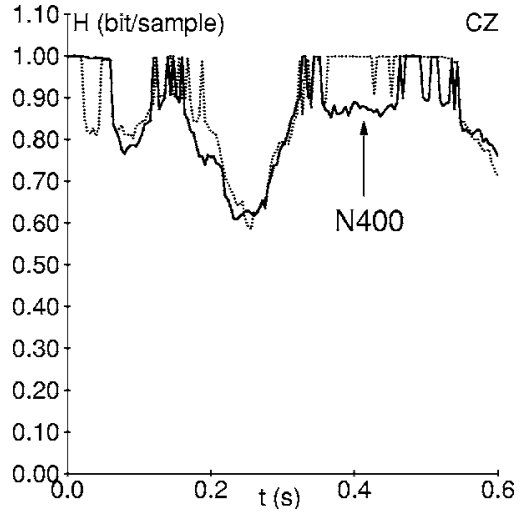

FIG. 4. Running cylinder entropy [Eq. (6)] of mean-field transformed three-word statistics for both conditions (ii) (solid) and (iii) (dotted) computed for the optimal encoding threshold $\theta^{\#}=4.2 \mu \mathrm{V}$ at channel CZ

$$
H^{\left(c_{j}\right)}(t)=-\sum_{i} p_{i}^{\prime\left(c_{j}\right)}(t) \log _{2} p_{i}^{\prime\left(c_{j}\right)}(t)
$$

of the mean-field filtered two-word statistics for the conditions $c_{1}, c_{2}$, respectively; which is presented for conditions (ii) and (iii) at $\theta^{\#}=4.2 \mu \mathrm{V}$ (see Fig. 4).

Finally, we average the cylinder entropies over the time window between onset $t_{\mathrm{on}}=300 \mathrm{~ms}$ and end $t_{\mathrm{off}}=600 \mathrm{~ms}$ of the N400 window of both conditions according to [[11], Eq. (3)], that has been used for the statistical analysis in [20]. From these time-averaged entropies

$$
G^{\left(c_{j}\right)}(\theta)=\frac{1}{t_{\mathrm{off}}-t_{\mathrm{on}}} \int_{t_{\mathrm{on}}}^{t_{\mathrm{off}}} H^{\left(c_{j}\right)}(t) d t,
$$

the SNR estimators

$$
S^{\left(c_{j}\right)}(\theta)=0.5883 \times\left(\frac{1}{G^{\left(c_{j}\right)}(\theta)}-1\right)
$$

are obtained. Figure 5 clearly demonstrates that the N400 ERP generates a stochastic resonance effect for both conditions (ii) and (iii). However, these resonance curves exhibit great differences which are not present in the ERP voltage averages (cf. Fig. 1). The resonance curve for condition (iii) assumes its maximum at a critical threshold $\theta_{\text {arg }}^{*}=4.7 \mu \mathrm{V}$, while the SNR curve for condition (ii) is maximal at $\theta_{\text {sem }}^{*}=4.4 \mu \mathrm{V}$. These critical thresholds might be identified with the "true" amplitudes of the ERP components. More important, however, is the maximal absolute difference of both resonance curves $S^{\left(c_{1}\right)}(\theta)$ and $S^{\left(c_{2}\right)}(\theta)$. The quantity

$$
q(\theta)=\left|S^{\left(c_{1}\right)}(\theta)-S^{\left(c_{2}\right)}(\theta)\right|
$$

serves as a test statistics for empirically obtained resonances. Its maximizing argument

$$
\theta^{\#}=\arg \max _{\theta} q(\theta)
$$

is the optimal threshold. In the present case illustrated by Fig. 5, for the optimal threshold at channel $\mathrm{CZ} \theta^{\#}=4.2 \mu \mathrm{V}$ the resonance effect is stronger in condition (ii) than in condition (iii).

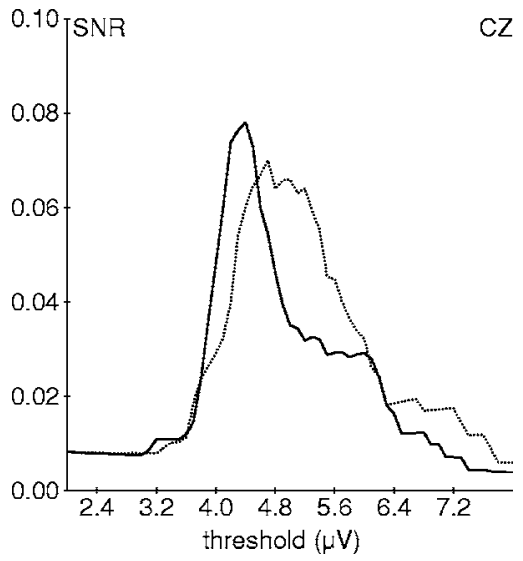

FIG. 5. Symbolically estimated signal-to-noise ratio (SNR, linear scale) [Eq. (8)] computed from running cylinder entropies averaged over the time window of the N400 ERP component from $300 \mathrm{~ms}$ up to $600 \mathrm{~ms}$ depending on the encoding threshold $\theta$ for both conditions (ii) (solid) and (iii) (dotted) at channel CZ exhibiting stochastic resonance. The optimal encoding threshold is given by the maximal absolute difference of both resonance curves: $\theta^{\#}=4.2 \mu \mathrm{V}$ [Eq. (10)].

To assess the statistical reliability of this effect, we have computed $M=10000$ replicas of a permutation test $[21,22]$ by exchanging approximately half of all symbolically encoded EEG epochs (i.e., 290) randomly between the grand epoch ensembles of both conditions. For each replica and for the original observation, we have determined the test statistics $q\left(\theta^{\#}\right)$ [Eq. (9)] at all electrodes whose rank numbers $R(q)$ yield the error probability of the first kind through

$$
p=\frac{M+1-R\left(q_{\mathrm{obs}}\right)}{M+1},
$$

where $R\left(q_{\text {obs }}\right)$ is the rank of the observed test statistics inserted into the set of replicas. For channel $\mathrm{CZ}$ we obtained $p=0.048$, which is significant at the level of $5 \%$ error probability.

\section{TOPOGRAPHIC BRAIN MAPPING}

Now we bring these results into a comprehensive representation by means of topographic brain mappings. Differences between the ERPs revealed by the SRA can be displayed either by maps of the critical thresholds $\theta_{k}^{*}$ (i.e., the threshold values for which the SNR is maximal), or by maps of the optimal thresholds $\theta_{k}^{\#}$ [Eq. (10)] (i.e., the threshold values where the differences across conditions are maximal), for each electrode $k$. Interestingly, for our experiment, we found no difference for the critical thresholds $\theta_{k}^{*}$ between the conditions (ii) and (iii), suggesting that the evoked brain responses are of almost the same amplitude. However, as Fig. 6(a) reveals, there are spatial differences in the optimal encoding thresholds $\theta_{k}^{\#}$, suggesting that there are differences in the resonance curves across the conditions. The contour line in Fig. 6(a) denotes the area where the result of the permutation test Eq. (11) is significant at the 5\% level. This map is very similar to that of the time-averaged voltage ERPs pre- 


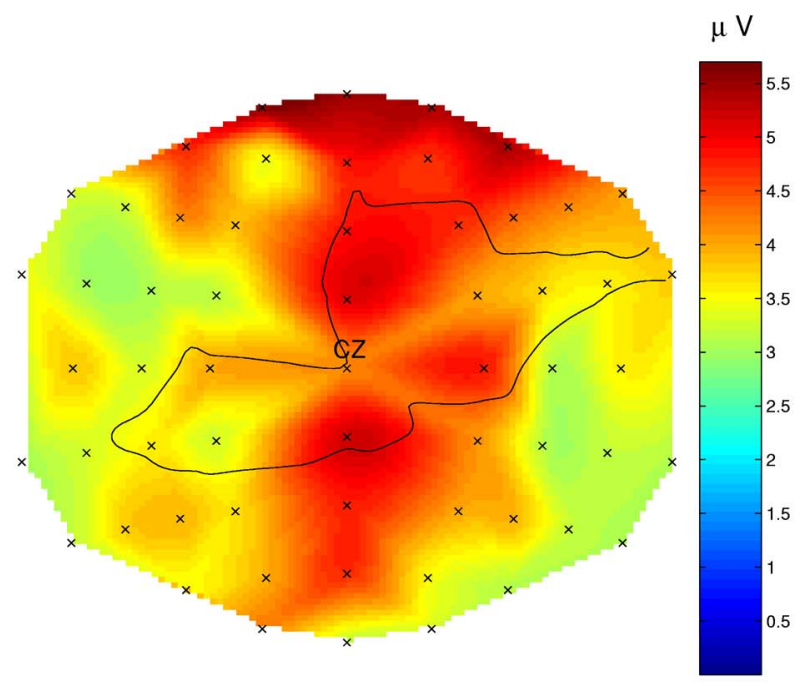

(a)

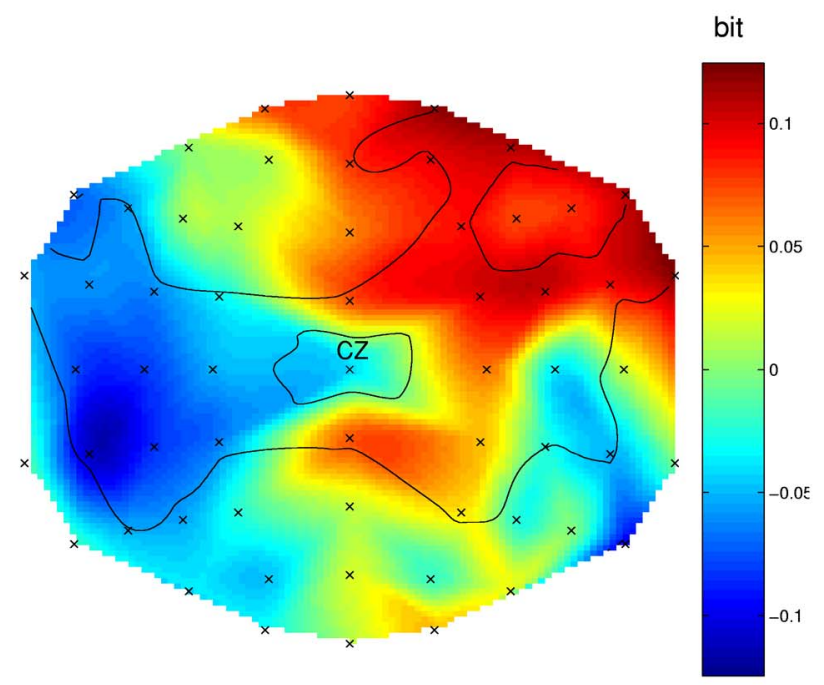

(b)

FIG. 6. (Color online) Topographic brain maps of (a) the optimal encoding thresholds $\theta^{\#}$ [Eq. (10)], and their corresponding significance of the permutation test Eq. (11) at the 5\% level shown by the contour. (b) Topographic brain map of the differences between the timeaveraged cylinder entropies Eq. (7), and their corresponding significance at the $5 \%$ level of a between-subject $t$-test shown by the contour. The nose is at the top. Additionally, the channel $\mathrm{CZ}$ is marked.

sented in Fig. 2, but in contrast to the averages, the permutation test shows that the difference between conditions (ii) and (iii) is now statistically significant at the central and temporal electrode sites.

While the plots of the optimal thresholds $\theta_{k}^{\#}$ shown in Fig. 6(a) are more related to the amplitude of the signal, the differences of the time-averaged cylinder entropies [Eq. (7)] plotted in Fig. 6(b) reflect the topographic differences in intertrial coherence between the conditions (ii) and (iii). In order to assess their statistical reliability, we had to decompose the grand epoch ensembles of all trials of all subjects into those contributed by each individual, respectively [17]. This can be achieved easily by replacing the grand ensemble word statistics $p_{i}^{\left(c_{j}\right)}(t)$ in Eq. (5) with their single-subject counterparts. The cylinder entropies thus obtained are then averaged and subjected to a $t$-test. The statistical significance for the $5 \%$ error probability is displayed in Fig. 6(b) by the contour lines. The coherence differences between the experimental conditions exhibit a dipolelike pattern where the coherence for the semantic violation condition (ii) is greater than that for the argument structure violation (iii) at leftparietal electrode sites, whereas it is smaller at right-frontal sites.

In summary, we find that the SRA is able to pull amplitude and coherence information apart, while the traditional voltage averages leaves these two kinds of information intermixed in one single dimension. In the SRA technique, voltage information is represented by the optimal encoding thresholds, whereas intertrial coherence (and hence phase information) is contained in the time dependent word statistics, the cylinder entropy and the SNR.

\section{DISCUSSION}

In this paper we employ the recently developed SRA technique [11] to EEG data from a language processing experi- ment [20]. In this experiment, the customary voltage averaging technique proves unable to distinguish between two N400 components in response to unacceptable sentence constructions. Such a difference, however, is expected on the basis of linguistic theory. Our method is based on nonlinear dynamics, namely, symbolic dynamics combined with a mean-field transformation, and describes ERP components as stochastic resonance effects. We find that the topographic representation of voltage and coherence information of the EEG data reveals a significant difference between the processing of a purely semantic violation compared to the processing of an argument structure violation.

The determination of the optimal thresholds compensates for the alleged loss of information entailed by the symbolic coarse-graining. The optimal thresholds contain all voltagerelated information of the ERP. In contrast to the voltage averaged ERP, where amplitude and phase information are mixed into one dimension, the SRA pulls the two kinds of information apart into two different dimensions: amplitude is represented by the critical encoding thresholds, whereas intertrial coherence is contained in the time-dependent word statistics and cylinder entropy. Furthermore, despite there being no difference across the critical thresholds in this experiment, we find optimal thresholds, showing a significant difference across the conditions. The fact that the permutation test shows that the optimal thresholds for particular electrodes differ significantly indicates that, despite little difference in amplitude, there are differences between the conditions, with the optimal thresholds defining those points where these differences, in the coherence and amplitude dimensions, are maximal.

A further advantage of the SRA against the averaging technique is that it is robust against outliers in the EEG, whereas the amplitudes of the voltage averaged ERP are very susceptible to them. Consider two cases: first, a possible out- 
lier is consistent with an ERP effect; i.e., the outlier has the same polarity as the ERP. By means of the coarse-graining, the outlier will then be represented by the very same symbol (either " 0 " or " 2 ") as the ERP, but contributing only one trial to the word statistics. On the other hand, in the averaging paradigm an outlier is weighted by its numerical value in the ERP average, which typically causes a large deviation. In the second case, when the outlier is not consistent with the ERP component, the ERP average is diminished by its numerical extend. Contrarily, one inconsistent outlier trial is treated by the mean-field transform of the SRA either as being undecided ("1") or as a loser in the competition of the meanfields. Thus, the coarse-graining of the SRA combined with the multiple-threshold analysis does not involve any renun- ciation of information, but is very robust against statistical outliers on the other hand.

Generally, the SRA seems to be promising for analyzing ensembles of noisy and nonstationary time series which are time-locked to certain events. This is the case especially in the neurosciences or in the geosciences.

\section{ACKNOWLEDGMENTS}

We gratefully acknowledge helpful discussions with Angela Friederici and we thank Carsten Allefeld for his support in preparing the brain maps. This work has been supported by the Deutsche Forschungsgemeinschaft within the research group on "Conflicting Rules in Cognitive Systems." A. F. was supported by the Fulbright Commission in Berlin.
[1] P. J. Brockwell and R. A. Davis, Time Series: Theory and Methods, Springer Series in Statistics, 2nd ed. (Springer, Berlin, 1991).

[2] H. Kantz and T. Schreiber, Nonlinear Time Series Analysis, Cambridge Nonlinear Science Series Vol. 7 (Cambridge University Press, Cambridge, UK, 1997).

[3] S. Makeig, M. Westerfield, T.-P. Jung, S. Enghoff, J. Townsend, E. Courchesne, and T. J. Sejnowski, Science 295, 690 (2002).

[4] R. Q. Quiroga, O. A. Rosso, E. Bașar, and M. Schürmann, Biol. Cybern. 84, 291 (2001).

[5] R. Q. Quiroga and E. L. J. M. van Luijtelaar, Int. J. Psychophysiol 43, 141 (2002).

[6] F. Moss, D. Pierson, and D. O'Gorman, Int. J. Bifurcation Chaos Appl. Sci. Eng. 4, 1383 (1994).

[7] L. Gammaitoni, P. Hänggi, P. Jung, and F. Marchesoni, Rev. Mod. Phys. 70, 223 (1998).

[8] T. Mori and S. Kai, Phys. Rev. Lett. 88, 218101 (2002).

[9] A. S. Pikovsky and J. Kurths, Phys. Rev. Lett. 78, 775 (1997).

[10] Z. Gingl, L. B. Kiss, and F. Moss, Europhys. Lett. 29, 191 (1995).

[11] P. beim Graben and J. Kurths, Phys. Rev. Lett. 90, 100602 (2003).
[12] E. Niedermeyer and F. L. da Silva, eds., Electroencephalography. Basic Principles, Clinical Applications, and Related Fields, 4th ed. (Lippincott Williams and Wilkins, Baltimore, 1999).

[13] J. Möcks, T. Gasser, and P. D. Tuan, Electroencephalogr. Clin. Neurophysiol. 57, 571 (1984).

[14] P. beim Graben, J. D. Saddy, M. Schlesewsky, and J. Kurths, Phys. Rev. E 62, 5518 (2000).

[15] C. Allefeld, S. Frisch, and M. Schlesewsky, NeuroReport 16, 13 (2004).

[16] M. Kutas and C. K. van Petten, in Handbook of Psycholinguistics, edited by M. A. Gernsbacher (Academic, San Diego, 1994), pp. 83-133.

[17] S. Frisch and P. beim Graben, Brain Res. Cognit. Brain Res. 24, 476 (2005).

[18] F. Y. Wu, Rev. Mod. Phys. 54, 235 (1982).

[19] P. beim Graben, Phys. Rev. E 64, 051104 (2001).

[20] A. D. Friederici and S. Frisch, J. Mem. Lang. 43, 476 (2000).

[21] P. Good, Permutation Tests, Springer Series in Statistics (Springer, New York, 1994).

[22] C. Allefeld and J. Kurths, Int. J. Bifurcation Chaos Appl. Sci. Eng. 14, 405 (2004). 